EXEMPLARIa Classica

Journal of Classical Philology

17, 2013, pp. 221-244

ISSN 1699-3225

\title{
SCHOLIA NON SERVIANA NEI MANOSCRITTI CAROLINGI DI VIRGILIO: PRIME NOTIZIE DEGLI SCAVI *
}

\author{
Silvia Ottaviano \\ Scuola Normale Superiore, Pisa \\ s.ottaviano@sns.it
}

\section{SUMMARY}

The author discusses $\mathbf{q}$, a fragmentary ms. of the mid-9th century from St Emmeram, Regensburg, in which the text of the $A e$ neid is accompanied by marginal scholia partly independent of Servius but related to some transmitted in Servius auctus. Close to them are those in another ms. of the same date, $\mathbf{x}$ (Montpellier H 253), and the first Vatican mythographer used material from the same source. Special attention has been paid to the glosses referring to an authority (e.g. Isidorus, Prudentius, Cicero), whose name sometimes is questionable (Columella, Linchis).

KEYWORDS

carolingian manuscripts of Virgil's works, Liber Glossarum, Mythographus Vaticanus, scholia non serviana, Servius, Servius Auctus

\section{RiASSUNTO}

L'autrice discute $\mathbf{q}$, un manoscritto frammentario della metà del IX sec. originario di S. Emmeram, Regensburg, in cui il testo dell'Eneide è accompagnato da scolii marginali in parte indipendenti da Servio ma connessi ad alcune note del Servius auctus. Simili a questi scolii sono quelli di un altro manoscritto coevo, x (Montpellier H 253), e anche il primo Mitografo Vaticano risulta aver impiegato materiali provenienti dalla stessa fonte. Una speciale attenzione è dedicata a quelle annotazioni che fanno menzione di un'autorità (es. Isidorus, Prudentius, Cicero), il cui nome a volte solleva dubbi (Columella, Linchis).

\section{PAROLE CHIAVE}

manoscritti carolingi delle opere di Virgilio, Liber Glossarum, Mythographus Vaticanus, scholia non serviana, Servius, Servius Auctus

Fecha de recepción: 20/08/2013

Fecha de aceptación y versión final: 28/09/2013

* Ringrazio gli anonimi referees di Exemplaria Classica, Ernesto Stagni e Veronika Von Büren per utili suggerimenti e correzioni. 
Nel 1974 Bernhard Bischoff individuò in alcuni frustuli, oggi dispersi nelle biblioteche di Monaco, Berlino e Bonn, i frammenti superstiti di un unico manoscritto di Virgilio (Aeneis cum scholiis), copiato nello scrittorio di S. Emmeram (Regensburg) intorno al secondo terzo del IX sec., come lo stesso Bischoff ha precisato più di recente ${ }^{1}$. I disiecta membra che componevano il codice originario ammontano complessivamente a 25 fogli, di cui restano in alcuni casi solo piccole parti:

-Berlin, Staatsbibliothek- Preussischer Kulturbesitz, Ms. lat. fol. $421: 12 \mathrm{ff}$. (Aen. 1.583-691; 2.57-167; 343-99; 512-89; 4.287-466; 6.577-820; 7.690-753)2;

-Bonn, Universitäts- und Landesbibliothek, S $476<$ fol. vv $>$ : frammento di pergamena contenente uno scolio (ad Aen. 6.288)

-München, Archäologische Staatssammlung, B. Starks Collectaneen Handschriften Nachlass, hist. Ver. 18, VIII: ff. 693-694 (Aen. 638-760)";

-München, Bayerische Staatsbibliothek, Clm 14105: foglio di guardia, ridotto ad una strisciolina $($ Aen. 11.109-41);

1 B. Bischoff, Die südostdeutschen Schreibschulen und Bibliotheken in der Karolingerzeit, I (Die bayerischen Diözesen), Wiesbaden 1974, 219-20; 271-2 (Tafel VII d); id., Katalog der festländischen Handschriften des neunten Jahrhunderts (mit Ausnahme der wisigotischen), II (Laon-Paderborn), Wiesbaden 2004, 276. Sui dati forniti da Bischoff si basa la descrizione del gruppo di frammenti presente in Munk Olsen, L'etude des auteurs classiques latins aux XI et XII e siècles, Paris 1985, II, 702-3; "Chronique des manuscrits classiques latins (IX ${ }^{\text {e-XII }}$ siècle)”, RHT 27, 1997, 79; RHT n.s., 2, 2007, 100.

${ }^{2}$ Il frammento fu acquisito dalla Staatsbibliothek probabilmente nel 1867 dall'antiquario Th. Kampffmeyer di Berlino: Ch. Meckelnborg, Handschriften klassischer lateinischer Autoren in der Staatsbibliothek Preussischer Kulturbesitz, Berlin 1986, 81. I margini di alcuni fogli sono stati tagliati con conseguenti perdite testuali: oltre a numerosi scolii, sono andati perduti i seguenti versi: Aen. 1.604-11 (f. 1r); 633-40 (1v); 663-9 (2r); 692-8 (2v); 2.81-7 (3r); 110-6 (3v); 139-45 (4r); 168-74 (4v); 7.688-9 (12r); 721-3 (12v).

${ }^{3} \mathrm{Il}$ frammento(insieme agli altri della stessa raccolta) fu acquisito dalla Universitätsbibliothek di Bonn nel 1834 dal giurista di Kiel A.W. Cramer. Inventariato originariamente con la segnatura S 251, in seguito fu indicato anche diversamente (S 183, 184, 186, 188, 476); a partire da 1980 è stato stabilito di citarlo esclusivamente con la segnatura $S 476$ e il numero di foglio (espresso con le lettere $a-z, a a-z z ; \alpha-\kappa)$.

4 Il frammento è contenuto nella raccolta dei manoscritti del padre benedettino Bernhard Stark, consistente in 21 volumi conservati fino al 1977 presso la Bibliothek des historischen Vereins fuer Oberbayern dello Stadtarchiv di Monaco e poi ceduti in prestito permanente alla Prähistorische (oggi Archäologische) Staatssammlung di Monaco. Ringrazio A. Löffelmeier per avermi segnalato questa circostanza, di cui non tiene conto il catalogo di Munk Olsen (e successivi aggiornamenti). Nessuna segnalazione in merito neppure in Mittelalterliche Bibliothekskataloge Deutschlands und der Schweiz, Band 4, Teil 1: Bistümer Passau und Regensburg. Bearb. von Ch. E. Ineichen-Eder, 1977, 139; S. Krämer, Handschriftenerbe des deutschen Mittelalters. Teil 1: Aachen - Kochel. Teil 2: Köln - Zyfflich (Mittelalterliche Bibliothekskataloge Deutschlands und der Schweiz, Ergänzungsband I, Teil 1 und 2. Im Auftrag der Bayerischen Akademie der Wissenschaften hrsg. v. B. Bischoff), 1989.

5 E. Wunderle, Katalog der lateinischen Handschriften der Bayerischen 
-München, Bayerische Staatsbibliothek, Clm 14361: foglio di guardia, ridotto ad una strisciolina (Aen. 6.821-49)6;

-München, Bayerische Staatsbibliothek, Clm 29216(8: 7 ff. (Aen. 2.287342; 590-646; 704-62; 3.305-62; 5.59-116; 234-91; 7.352-423)

-München, Bayerische Staatsbibliothek, Clm 29216(20: frammento di pergamena contenente parti di due scolii (ad Aen. 3.402; 3.405) .

Rispetto alle indicazioni fornite da Bischoff (ripetute senza modifiche nei cataloghi successivi) alcune segnature sono cambiate, come ho avuto modo di constatare io stessa contattando le singole collezioni per verificare la corrispondenza con le segnature attuali ${ }^{9}$. I frammenti presentano numerosi aspetti interessanti: in primo luogo sono originari di uno scrittorio decentrato rispetto al nord-est della Francia, regione che a giudicare dai manoscritti conservati può esser considerata il crogiolo della filologia virgiliana nel IX sec. A ciò si aggiunge che la mano responsabile di tutti gli scolii impiega una scrittura molto caratteristica (tanto da rendere inequivocabile l'attribuzione dei frammenti allo stesso codice originario), dai tratti marcatamente insulari. In realtà nessuno di questi due elementi stupisce: S. Emmeram, come altri scrittori della Germania meridionale, nel IX sec. era interessato da fitte relazioni con la Neustria, dove la presenza di Scotti (così come, in generale, nell'Occidente carolingio) è ben documentata ${ }^{10}$.

Staatsbibliothek München. Die Handschriften aus St. Emmeram in Regensburg, Bd. 1 (Clm 14000-14130), Wiesbaden 1995, 257. Si tratta di un foglio tagliato longitudinalmente (ne resta solo una strisciolina delle dimensioni di $31 \times 3.5 \mathrm{~cm}$ ) posizionato prima del foglio di guardia del Clm 14105. Nel recto contiene i versi (mutili della parte finale) di Aen. 11.109-41 (33 vv. per pagina).

${ }^{6}$ Nessuna segnalazione in F. Helmer, H. Hauke, E. Wunderle, Katalog der lateinischen Handschriften der Bayerischen Staatsbibliothek München: Die Handschriften aus St. Emmeram in Regensburg. Bd. 3: Clm 14261-14400, Wiesbaden 2011, 28. Come per il frammento precedente, anche in questo caso sopravvive un sottile frustulo contenente nel recto solo alcune lettere delle prime parole di ogni verso (presumibilmente il verso del foglio conteneva Aen. 6.850-79).

${ }^{7}$ H. Hauke, Katalog der lateinischen Fragmente der Bayerischen Staatsbibliothek München (Catalogus codicum manu scriptorum Bibliothecae Monacensis; T. 4, Ps. 12,1), Bd. 1 (Clm 29202-29311), Wiesbaden 1994, 30-1; 35. Riproduzione consultabile online all'indirizzo: http://bsb-mdz12-spiegel.bsb.lrz.de/ db/0007/bsb00071106/images/

${ }^{8}$ Hauke, Katalog, 35. Riproduzione consultabile online all'indirizzo: http://bsb-mdz12spiegel.bsb.lrz.de/ db/0007/bsb00071118/images/

9 Bischoff segnalava anche due frustuli conservati come fogli di guardia nel Clm $13581 \mathrm{e}$ nel Clm 14384; oggi tuttavia non vi è traccia di alcun frammento virgiliano in questi due codici: probabilmente questo materiale è confluito nel Clm 29216(8, visto che il numero di fogli superstiti noti a Bischoff $(23+2$ foglietti contenenti scolii) è rimasto invariato.

${ }^{10} \mathrm{~J}$. J. Contreni, The Irish in the Western Carolingian Empire, in Die Iren und Europa im früheren Mittelalter (ed. H. Löwe), Stuttgart 1982, II, 758-98; R. McKitterick, The diffusion of insular culture in Neustria between 650 and 850: the implications of the manuscript evidence, in La Neustrie. Les pays au nord de la Loire de 650 a 850 (ed. 
Il contenuto degli scolii conferma la particolare provenienza di questo testimone virgiliano: ho constatato infatti che la maggior parte delle annotazioni marginali coincide con quelle presenti in un altro codice carolingio di Virgilio, Montpellier, Bibliothèque interuniversitaire, Section Médecine, H 253 (secondo terzo del IX sec., copiato nel nord-est della Francia secondo Bischoff ${ }^{11}$, siglum $\mathbf{x}$ in Conte 2009 e Ottaviano-Conte 2013 ${ }^{12}$ ). Si tratta di note per lo più di carattere storico-mitologico che spesso presentano materiali non serviani ${ }^{13}$ : perciò ho ritenuto opportuno farne oggetto di un'indagine approfondita, collazionando personalmente testo e scolii dei frammenti indicati da Bischoff (di cui, a parte il frammento conservato a Bonn, ho consultato gli originali).

L'importanza di questo testimone, ben verificabile nonostante la sua incompletezza, mi ha inoltre spinto a includerlo nella recensio stabilita in accordo con G. B. Conte per la nuova edizione teubneriana dell'Eneide, da cui sono invece stati esclusi i frammenti minori del IX sec.; il siglum ivi utilizzato (q) per comodità verrà citato anche nel seguito, con riferimento sia al testo che agli scolii (e lo stesso valga per le sigle degli altri manoscritti virgiliani citati).

Il presente lavoro è inteso a fornire i principali risultati di tale ricerca in vista di uno studio più esaustivo sull'argomento ${ }^{14}$, attraverso una sintesi ragionata che proponga le prime impressioni e gli interrogativi che suscitano questi testi.

Per cominciare, mettendo insieme i frammenti oggi conservati separatamente è possibile ricostruire, almeno in parte, la struttura interna del codice originario: esso doveva constare principalmente di binioni e quaternioni e ogni pagina doveva contenere $28-35$ versi: il numero di righi cambiava anche all'interno dei singoli libri, a quanto pare spesso in corrispondenza del passaggio da un fascicolo all'altro. In particolare, si possono agevolmente ricostruire i quaternioni contenenti Aen. 2.228-703 (ff. I, IV, VIII perduti), Aen. 2.704-3.362 (ff. II-VII perduti); Aen. 4.166-647 (ff. I, II, VI-VIII perduti); Aen. 4.648-5.412 (ff. I, II, IV, V, VII, VIII perduti); Aen. 6.456-7.9 (ff. I, II, VII, VIII perduti).

Hartmut Atsma), Beihefte der Francia 16/II, Sigmaringen 1989, 395-432 (= R. McKitterick, Books, scribes and learning in the Frankish kingdoms, sixth to ninth centuries, Aldershot 1994, Ch. 3).

${ }^{11}$ Munk Olsen, L'etude, 737-8. Si veda più di recente la breve presentazione del manoscritto in P. Vergilius Maro, Aeneis, edidit G. B. Conte, Berlin/New York 2009, XXIV. Sto preparando una descrizione di questo manoscritto basata sulle ricerche che ho condotto negli ultimi anni sul testo e sugli scolii.

${ }_{12}$ P. Vergilius Maro, Bucolica et Georgica, ed. S. Ottaviano, G. B. Conte, Berlin/New York 2013.

13 Questo aspetto non è stato finora segnalato. Bischoff, Die südostdeutschen Schreibschulen, 219, 271 rinviava semplicemente a Servio.

${ }^{14}$ Una descrizione dei frammenti con trascrizione completa degli scolii è in preparazione per il sito Manuscripta Vergiliana, che sarà accessibile a breve dalla pagina: http://www.sns. it/ricerca/lettere/manoscrittivirgilio/ 
Un dato interessante che emerge da questa ricostruzione è che, almeno fino al quinto libro, il manoscritto originario probabilmente non conteneva gli Argumenta ai singoli libri dell' Eneide ${ }^{15}$. L'impaginazione del manoscritto non è, come in genere avviene, speculare: il testo è scritto nella parte sinistra della pagina, in modo da lasciare libero ora il margine esterno (nel recto), ora quello interno (nel verso) per gli scolii. Il frammento di Bonn e il Clm 29216(20 pongono un interrogativo circa la loro collocazione nel manoscritto: in entrambi i casi un lato del foglio non contiene testo e le dimensioni sembrano difficilmente conciliabili con quelle dei margini di una pagina contenente anche i versi di Virgilio ${ }^{16}$, a meno di non supporre che i margini superiore e/o inferiore fossero molto più ampi e siano stati in seguito rifilati. Perciò Hauke ipotizzava per il frammento monacense che si trattasse di un foglio isolato contenente esclusivamente scolii aggiunto nel manoscritto originario. Ciò troverebbe un parallelo nel Montp. H 253, dove un foglio isolato (il f. 120, contenente due colonne di scolii, come il Clm 29216(20) è stato inserito in un fascicolo a mo' di mantissa, evidentemente per rimediare alla mancanza di spazio sufficiente nei margini.

Per quanto riguarda le mani attive nel codice, Bischoff ${ }^{7}$ ha individuato un'unica mano fino al quarto libro dell'Eneide, appartenente ad un gruppo di manoscritti copiati nello scrittorio di S. Emmeram caratterizzati, oltre che dalla forma delle lettere e dal ductus, dalle legature $r q$ e $s p$ e dall'impiego saltuario delle abbreviazioni insulari per est, post, trans, con/cum, autem.

Una mano contemporanea è responsabile delle glosse e degli scolii in tutto il manoscritto e copia anche il testo a partire dal quinto libro e almeno fino al settimo libro ${ }^{18}$. La scrittura di questa mano, definita da Bischoff una 'keltische Minuskel', presenta più spiccati tratti insulari ${ }^{19}$, riscontrabili non solo nell'impiego sistematico di abbreviazioni caratteristiche, ma anche nella forma delle lettere, per quanto alcuni elementi (come la forma della $r$ e della $s$, o il legamento ro) si avvicinino alle scritture continentali. A ciò si

${ }^{15}$ In realtà in nessun caso è conservato l'incipit di un libro; tuttavia dal computo dei versi contenuti in ciascuna pagina restano esclusi i decastici prefatori, che comunque potrebbero esser stati aggiunti in margine.

${ }^{16} \mathrm{Il}$ frammento Clm 29216(20 misura 6.3 x $21.3 \mathrm{~cm}$ (Hauke, cit., 35); il frammento di Bonn misura $6.5 \times 16.5 \mathrm{~cm}$. Entrambi i frammenti contengono 12 righe di scolii, disposti su due colonne nel caso del Clm 29216(20. I fogli contenuti negli altri frammenti sono stati variamente tagliati, ma l'altezza massima è di $32 \mathrm{~cm}$ e la larghezza massima di $20.3 \mathrm{~cm}$ : con uno specchio di rigatura di $23-25 \times 15 \mathrm{~cm}$, i margini superiore e inferiore non superano 4 e $5 \mathrm{~cm}$ rispettivamente.

${ }^{17}$ Bischoff, Die südostdeutschen Schreibschulen, 219-20.

${ }^{18}$ Il testo dell'undicesimo libro copiato nel frammento Clm 14105 presenta una scrittura molto simile a quella della mano responsabile dei primi quattro libri; un ulteriore sintomo di continuità si ricava dalla presenza di iniziali maiuscole, usate solo nei primi quattro libri e nel frammento del libro undicesimo.

${ }^{19}$ Tra le abbreviazioni elencate da Bischoff si segnala in particolare quella per est, realizzata con un tratto orizzontale con un punto sopra e una virgola (o, più raramente, un punto) sotto. 
aggiunge che Bischoff ha identificato questa mano con quella attiva in un manoscritto coevo dell'Ars di Consenzio (Clm 14666), che a suo parere fu confezionato altrove (presenta infatti un sistema di rigatura tipicamente insulare) e portato (e forse anche copiato) a S. Emmeram. In definitiva, per lo studioso si tratterebbe di uno scriba originario del Galles o della Cornovaglia: un fenomeno alquanto raro nella casistica dei codici copiati nel continente nel IX sec. ${ }^{20}$.

Il testo di Virgilio tràdito da $\mathbf{q}$ sembra confermare la specialità di questo testimone: in alcuni casi, a differenza di quasi tutti gli altri manoscritti contemporanei, q conserva una lezione giusta, e.g. Aen. 4.436 dederit] dederis $\omega$ (praeter pqwy); in altri punti $\mathbf{q}$ conferma delle varianti forse giuste, e.g. Aen. 6.718 quo magis Italia mecum laetere reperta (italiam... repertam FRq) ${ }^{21}$; Aen. 7.392 furiisque accensas pectore matres (pectora cqtw, coni. Bentley). Altrove q attesta una variante piuttosto rara, e.g. Aen. 4.390 parantem] volentem Mcq; Aen. 4.433 furori] furoris pq Char. 547.55; Aen. 4.436 cumulatam] cumulata $\mathrm{M}_{5} \mathrm{~b}$ ?qw, Lib. Gloss. CU 158-9; Aen. 6.597 obunco] abunco FRctq.

Dall'esame degli errori singolari di q emerge la tendenza del copista all'inversione di parole (e.g. Aen. 6.675: vos si] si vos; Aen. 6.778: viden ut geminae] ut geminae vides), o all'eco di loci similes (e.g. Aen. 6.716: memorare] enumerare, cf. v. 717; Aen. 6.679: conualle] inualle, cf. georg. 2.488; la variante si trova anche in Anth. Lat. 1 VI 8 R.).

Ma è il testo degli scolii ad offrire le novità più suggestive, come si è già anticipato. Essenzialmente mi sembra che si possano distinguere due principali tipologie: scolii interamente ricavati dal commento di Servio (in genere a partire dalla versione aucta, con una rielaborazione formale più o meno vistosa) e scolii che, pur presentando a volte analogie con il testo di Servio, contengono materiali diversi e, almeno in parte, forse antichi. Come si è detto, alcuni degli scolii di $\mathbf{q}$ si ritrovano anche in $\mathbf{x}$, e a volte presentano forti analogie con il primo Mythographus Vaticanus, che mostra di aver attinto in molti casi a materiale esegetico virgiliano non serviano ${ }^{22}$.

Per cominciare, si veda un caso in cui si realizza un discreto accordo tra i testimoni (q, x, Servius auctus, Myth. Vat.). Ecco il testo (secondo la mia

${ }^{20}$ B. Bischoff, Irische Schreiber im Karolingerreich, in Jean Scot Érigène et l'histoire de la philosophie, cur. R. Roques, Paris 1977, 47-58 (= Mittelalterliche Studien, Stuttgart 1981, III, 39-54, spec. 40 n. 4; 47 n. 53).

${ }^{21} \mathrm{Si}$ vedano i casi in cui laetor regge un'infinitiva in cui è sottinteso il verbo esse elencati in ThLL VII-2 c. 881, 5s. (e.g. Cic. Fin. 5.96: laetorque eam philosophiam...hanc igitur laetor etiam acutiorem repertam quam ceteras; Sall. Iug. 110. 3: id inminutum quod ceteri dolere solent ego laetor; Liv. 30.30.3).

${ }^{22}$ Per la datazione dell'opera (875-1075), e la questione delle fonti si veda: Le premier mythographe du Vatican, Paris 1995, ed. N. Zorzetti, trad. J. Berlioz, XI ss. Il Mythographus è tràdito dal Reg.lat.1401, manoscritto della fine del XII sec. 
ricostruzione ${ }^{23}$ ) dello scolio $\mathbf{q x}$ relativo al personaggio di Teucro, menzionato ad Aen. 1.619 (Teucrum...Sidona venire):

Teucer Telamonis ${ }^{1}$ filius et Hesionae ${ }^{2}$ filiae Laomedontis quam Hercules secum adduxit cum expugnasset Troiam, Laomedonte interfecto, et comiti suo Telamoni ${ }^{3}$, qui ${ }^{4}$ primus murum ascendit, in matrimonium dedit; ex qua Telamon ${ }^{5}$ duos filios habuit, Teucrum et Aiacem $^{6}$. Is ${ }^{7}$ autem Teucer quia ${ }^{8}$ non dedit auxilium fratri suo interfecto apud Troiam et quod ossa eius in patriam non retulerit et filium parvulum eius, Eurysacem ${ }^{9}$ nomine $^{10}$, secum ${ }^{11}$ non adduxerit credebatur $\left\langle\right.$ eum $>^{12}$ occidisse ne coheres eius esset. Actus a patre in exilium Belum Phoenicum ${ }^{13}$ regem adiit, qui Cyprum insulam armis compulerat $^{14}$; ibi Teucer ${ }^{15}$ commisso bello ${ }^{16}$ urbem condidit, quam Salaminam ${ }^{17}$ nomine patriae ${ }^{18}$ nominavit ${ }^{19}$. Teucer inter hostes Danaos fuit; alius est Teucrus a quo Troiani.

1 talamonis $\mathbf{q} \mid 2$ isionae $\mathbf{x} \mid 3$ talamoni $\mathrm{q} \mid 4$ qui...ascendit] om. $\mathrm{q} \mid 5$ thalamo $\mathrm{q} \mid 6$ adiacem $\mathbf{q}$ ad aiacem $\mathbf{x} \mid 7$ is] iste $\mathbf{q}$ his $\mathbf{x}^{\text {acl }} 8$ qui $\mathbf{x} \mid 9$ Eurysacem] $c f . D S$ a.l. iorizacem x om. q | 10 nomine] om. $\mathbf{q} \mid 11$ secum...esset] om. $\mathbf{x} \mid 12$ eum supplevi, cl. Myth. Vat. I 2.36.2 $\mid 13$ poenicum $\mathbf{x} \mid 14$ compulerat] scripsi, cl. Myth. Vat. (subegerat) compellebat q compellerat $\mathbf{x} \mid 15$ teucer] om. $\mathbf{x} \mid 16$ comissu beli $\mathbf{x} \mid 17$ Salaminam] scripsi, cl. DS ad Aen.1.622 Talamoniam q salomoniam x| 18 patriae] scripsi, cl. DS, Myth. Vat. patris sui q patriae matris Acidaliae $\mathbf{x} \mid 19$ quae modo nuncupatur consta $<\mathrm{n}>\mathrm{tia} a d d$. $\mathbf{x}$

Si confronti il commento del Servius auctus relativo allo stesso passo (le aggiunte, segnalate d'ora in poi con l'abbreviazione DS, sono riportate in corsivo, come nell'edizione Thilo-Hagen ${ }^{24}$ ):

Serv. ad Aen. 1.619 TEVCRVM MEMINI SIDONA VENIRE... a cuius portu cum eum Laomedon arceret, occisus est, et eius filia Hesiona belli iure sublata comiti Telamoni tradita est, qui primus ascenderat murum, unde Teucer natus est; nam Aiacem ex alia constat esse procreatum. tunc Hercules Priamum quoque redemptum a vicinis hostibus in paterno regno locavit: unde et Priamus dictus est ஷ̀đ ceterum quae de liberata dicuntur Hesiona constat esse fabulosa. Sed

${ }^{23} \mathrm{Nel}$ presentare l'edizione provvisoria di alcuni scolii dei manoscritti q e $\mathbf{x}$ ho cercato di risalire, ove possibile, allo stadio più antico della loro redazione, sulla base di confronti con altri autori. Ne risulta un testo ibrido che non ha alcuna pretesa di riprodurre un testo reale ma costituisce un tentativo di render conto delle varie fasi di formazione degli scolii attuali a partire da un nucleo antico.

${ }^{24}$ Per uniformità cito tutti i passi di Servio da Servii Grammatici qui feruntur in Vergilii carmina commentarii, ed. G. Thilo, Lipsiae 1881-1887. Ho segnalato le discrepanze rispetto alle edizioni parziali più recenti laddove fossero significative per il confronto con gli scolii di qx in questione (di cui finora non ha tenuto conto nessuna edizione). 
Teucer cum Troia eversa sine fratre esset reversus, qui se furore propter perdita Achillis arma interemerat, vel, ut quidam volunt, quia non defenderat Aiacis fratris interitum, ut alii, quod ossa fratris non rettulisset, ut nonnulli, quod Tecmessam concubinam vel eius fratris filium Eurysacen ad avum Telamona de Troia secum non reduxerit, quia ille alia navi vectus felicius navigaverat, Salamine pulsus a patre Sidona venit, ex quo Dido cuncta cognovit. Aiax autem Achillis patruelis frater fuerat, quoniam Telamon et Peleus fratres fuerunt, Aeaci filii.

Ad Aen. 1.622 VASTABAT CYPRVM quam subactam concessit Teucro, ut in ea conlocaret imperium, qui ex responso Apollinis illuc perrexerat et civitatem ${ }^{25}$ Salaminam ex nomine patriae ibi condidit, licet alii dicant ab ipso Teucro Cypri superatos incolas et sic conditam civitatem: unde 'auxilio Beli' accipi potest, etiam Teucro auxilium a Belo ad occupationem insulae praestitum.

Come si può subito osservare, lo scolio $\mathbf{q x}$ presenta punti di contatto notevoli quasi esclusivamente con le aggiunte DS. Infatti, il nostro scolio quasi non trova alcuna corrispondenza con la prima parte del commento serviano relativa alle imprese troiane di Ercole (qui omessa per brevità: cuius portu si riferisce a Troia, dove Ercole arrivò dopo la perdita di Ila; eum = Herculem $)$, a parte il segmento relativo all'uccisione di Laomedonte e all'unione tra Esione e Telamone (comiti Telamoni tradita est, qui primus ascenderat murum comiti suo Telamoni, qui primus murum ascendit, in matrimonium dedit); si noti tuttavia la vistosa divergenza tra lo scolio e Servio in relazione alla parentela tra Aiace e Teucro: Servio, evidentemente in polemica con un altro commentatore da cui forse discende il nostro scolio, si peritava di precisare che i due eroi, entrambi figli di Telamone, non fossero nati dalla stessa madre.

La seconda parte dello scolio (is autem Teucer...secum non adduxerit), si avvicina molto al testo DS, sia nella sostanza che nella ripresa di alcune espressioni (quod ossa fratris non rettulisset quod ossa eius in patriam non retulerit; eius fratris filium Eurysacen...secum non reduxerit filium parvulum eius, Eurysacem nomine, secum non adduxerit). Va tuttavia notato che mentre DS distingue le varie ragioni addotte per spiegare perché Teucro facesse ritorno senza Aiace, attribuendole a fonti diverse ( $u t$ quidam volunt...ut alii...ut nonnulli), nello scolio non vi è traccia di tale distinzione: con qualche semplificazione (è omesso il particolare relativo a Tecmessa) le tre versioni sono presentate simultaneamente come motivazioni

${ }^{25}$ In luogo del testo così ricostruito da Thilo i codici del Servius Auctus hanno: Teucro ex responso Apollinis illuc perrexerat ut in ea conlocaret imperium, qui eam civitatem etc. 
dell'accusa rivolta a Teucro di aver ucciso il fratello per restare erede unico: un'altra vistosa differenza rispetto a Servio, che invece si limita a seguire la versione tradizionale del suicidio di Aiace. In realtà tra il testo di Servio e l'aggiunta DS si nota una certa incongruenza, per cui sorge il sospetto che lo scolio qx rimonti al testo stesso da cui fu tratta l'aggiunta DS (in altre parole, il segmento credebatur eum occidisse ne coheres eius esset sarebbe antico).

La parte finale dello scolio fonde la nota serviana a Aen. 1.619 (nella versione DS: pulsus a patre actus a patre in exilium) con la nota DS a 1.622, volta a fornire notizie circa la fondazione di Salamina da parte di Teucro (civitatem Salaminam ex nomine patriae ibi condidit $\sim$ urbem condidit, quam Salaminam nomine patriae nominavit). Anche in questo caso lo scolio segue la versione alternativa rispetto a Servio fornita da DS, secondo cui Teucro avrebbe combattuto per impossessarsi di Salamina (ibi Teucer commisso bello urbem condidit: cf. DS licet alii dicant ab ipso Teucro Cypri superatos incolas et sic conditam civitatem), avvalendosi a tal scopo dell'aiuto di Belo (cf. DS Teucro auxilium a Belo ad occupationem insulae praestitum; forse così va interpretata la variante di $\mathbf{x}$ commissu Beli tràdita da in luogo di commisso bello, confermato dalla lezione longo bello del Myth. Vat., v. infra).

Infine, l'osservazione conclusiva (hic autem Teucer...Teucrus a quo Troiani) mostra una chiara matrice scolastica e va forse ascritta al compilatore medievale, particolarmente interessato alla genealogia di Dardano e all'origo Troianorum.

Anche il confronto con il Mythographus Vaticanus si dimostra molto interessante, perché una parte del testo coincide esattamente con il nostro scolio, che va perciò avvicinato alle fonti utilizzate dal Mitografo. Si legga dunque (secondo l'edizione di Zorzetti ${ }^{26}$, cui si rinvia anche nel seguito):

Myth. Vat. I.2.36. 1. Teucer cum Troia spoliata sine fratre esset reversus, qui se furore propter arma perdita Achillis interemerat, Salamine pulsus Sidonem venit; ex quo Dido cuncta[m] cognovit. 2. Is Teucer quod auxilium non dedit fratri suo interfecto -ut quidam volunt- apud Troiam et quod ossa eius in patriam non detulerit et filium eius parvulum secum non adduxerit, credebatur eum occidisse ne coheres eius esset. 3. Actus a patre in exilium, Belum, Phoenicum regem, adiit, qui Cyprum insulam armis subegerat; ibi Teucer longo bello urbem condidit, quam Salami<n>am nomine patriae vocavit. 4 .

${ }^{26}$ Zorzetti, Le premier mythographe, 79, n. 407, indica come fonte per i parr. 1 e 5 lo scolio serviano a Aen. 1.619; per il par. 4 Aen. 3.104 con il relativo commento serviano, mentre per i paragrafi 2-3 lo studioso, pur rimandando al testo del Servius auctus ad Aen. 1.619, riteneva che la fonte diretta del Mitografo fosse uno scolio sconosciuto, da identificare con il nostro. 
Hic Teucer cum hostibus Danais ${ }^{27}$ fuit; alius est Teucrus a quo Troiani Teucri dicuntur, qui post Dardani obitum Troiae muros exstruxit et ampliavit. 5. Aiax autem et Achilles patrueles fuerunt, quoniam Telamon et Peleus fuerunt fratres, Aeaci filii.

Due sono le principali differenze tra il testo del Mitografo e lo scolio in questione: il Mitografo contiene un riferimento alle notae variorum maldestramente desunto da DS (ut quidam volunt) 28 $^{28}$ aggiunge un segmento di testo relativamente a Teucro e Dardano (Teucri dicuntur... ampliavit).

In quest'ultimo caso Zorzetti giustamente confronta Serv. ad Aen. 3.104 «CRETA Dardanus Iovis filius et Electrae, profectus de Corytho, civitate Tusciae, primus venit ad Troiam et illic parva aedificia collocavit. Post cuius obitum Teucer venit ex Creta et invenit Dardani socios habitantes in vallibus, qui constituit arces et moenia». $\grave{E}$ dunque verosimile che la discrepanza sia dovuta ad un'innovazione del Mitografo. Lo stesso si può dire per longo bello in luogo di commisso bello e qualche altro dettaglio (subegerat in luogo di compulerat; detulerit in luogo di retulerit).

Più delicato appare il problema della citazione da DS (ut quidam volunt): si dovrebbe pensare a collazione con il Servius Auctus oppure (più verosimilmente) alla sopravvivenza di questo segmento testuale nell'esemplare stesso da cui proviene lo scolio qx, magari sotto forma di aggiunta marginale: ciò spiegherebbe da un lato l'omissione da parte di q e $\mathbf{x}$, dall'altro la collocazione inadeguata dell'inciso nel testo del Mitografo.

In ogni caso, come emergerà con più chiarezza nel seguito, il Mitografo mostra di conoscere una versione più simile a quella tràdita da q piuttosto che da $\mathbf{x}$ (si veda, ad esempio, l'omissione del nome di Eurisace, comune a $\mathbf{q}$ e al Mitografo).

Infine, per quanto riguarda le varianti di $\mathbf{q}$ e $\mathbf{x}$ e la ricostruzione del testo da cui i due scolii derivano, si può osservare che i due manoscritti sono fra loro indipendenti, dal momento che ciascuno presenta errori singolari differenti (in particolare due cospicue omissioni); l'unico errore congiuntivo è adiacem / ad aiacem per Aiacem, ma in altri casi la messe è più abbondante, a conferma ulteriore dell'ipotesi di un archetipo comune.

Venendo al testo dello scolio in questione, per ricostruirne la forma originaria possiamo utilmente avvalerci del confronto con il Servius auctus e

${ }^{27}$ Danais è congettura di Mai (in luogo del tràdito Danaum), confermata da Danaos nello scolio dei manoscritti qx.

${ }^{28}$ Zorzetti, Le premier mythographe, 79, a proposito dei connettivi quod...ut quidam volunt...et quod...et, commenta in apparato: «haec male abbreviata potius quam corrupta mihi videntur». Forse la collocazione di ut quidam volunt dopo interfecto è condizionata dalla versione relativa all'assassinio di Aiace da parte di Teucro (credebatur eum occidisse), assente in DS e comune allo scolio qx e al Mitografo. 
con il Mythographus Vaticanus ${ }^{29}$. Ad esempio, non vi è dubbio che si debba scrivere: Salaminam nomine patriae nominavit, nonostante in questo punto sia $\mathbf{q}$ che $\mathbf{x}$ presentino un testo differente: Talamoniam [sic!] nomine patris sui nominavit (q) e Salomoniam [sic!] nomine patriae matris Acidaliae nominavit, quae modo nuncupatur Constantia (x). Per quanto riguarda q, mi pare possibile che, a seguito della corruzione di salaminam in salamoniam (cf. salomoniam in $\mathbf{x}$ ), il testo sia stato ritoccato da un copista ingegnoso in Talamoniam (= Telamoniam: in q il nome Telamon è costantemente alterato in Talamon): di conseguenza patriae sarebbe stato modificato in patris sui. In $\mathbf{x}$ invece colpisce l'annotazione quae modo nuncupatur Constantia, che si riferisce al nuovo nome assegnato a Salamina di Cipro in onore di Costanzo II (RE s.v. Constantia, cl. 953), particolare ricordato sia da Gerolamo (Epist. 108: Epiphanius Salaminae Cypri, quae nunc Constantia dicitur), che da Beda (Nom. loc. in Act. Ap., Migne L. 92, 1039: Salamis...nunc Constantia dicta). Quest'aggiunta dunque presuppone un testo corretto (Salaminam) e perciò a mio parere discende direttamente dall'archetipo dello scolio qx; ho scelto tuttavia di non metterla a testo ma solo in apparato perché credo si tratti di una glossa recenzione (anche se più antica di q e x!) annotata accanto allo scolio antico, che era mia premura restituire in una forma il più possibile vicina a quella originaria. Invece la glossa matris Acidaliae non sembra aver attinenza con il contesto e probabilmente va considerato un lemma (Aen. 1.720) annotato a margine nell'antigrafo di $\mathbf{x}$ e penetrato a testo per errore.

Un caso ancor più complesso e interessante è fornito dalla nota ad Aen. 6.288, tràdita da $\mathrm{q}$ (nel frammento conservato a Bonn) in una forma diversa $\mathrm{e}$ più ampia rispetto a $\mathbf{x}$; ecco i due scolii in edizione sinottica (la parte comune è riportata una sola volta):

${ }^{29}$ In apparato cito il Mitografo in tre casi: per l'integrazione di eum (in una sezione omessa da $\mathbf{x}$ ), per la correzione compulerat e per Salaminam: nel primo caso credo che il Mitografo preservi effettivamente il testo dello scolio originario, nel secondo caso credo che lo rifletta implicitamente ( pur variando il verbo), nel terzo caso credo che abbia emendato il testo corrotto dell'antigrafo (salamoniam?) facendo ricorso a Servio. 


\begin{tabular}{|c|c|}
\hline $\mathrm{q}$ & $\mathrm{x}$ \\
\hline \multicolumn{2}{|c|}{ Chimera monstrum fuit ${ }^{1}$, Typhonis ${ }^{2}$ et Echidnae ${ }^{3}$ filia, } \\
\hline $\begin{array}{l}\text { cuius triplex erat forma: prima facie } \\
\text { leo, media caprea flagrabat igni, cauda } \\
\text { minabatur ut draco. }\end{array}$ & $\begin{array}{l}\text { quod primas partes leonis, sequentes } \\
\text { caprae vomentis ignem, ultimas in } \\
\text { cauda serpentis habuit. }\end{array}$ \\
\hline \multicolumn{2}{|c|}{ Haec in Lycia ${ }^{4}$ iuxta Cragum ${ }^{5}$ montem populabatur terras. } \\
\hline $\begin{array}{l}\text { Bellerophons }{ }^{6} \text { autem cum admissa } \\
\text { c<a>ede patria profugus pervenisset ad } \\
\text { Proetum }^{8} \text { Argivorum }{ }^{9} \text { regem suscep- } \\
\text { tusque esset hospitio, adamatus est ab } \\
\text { uxore eius Stheneboea }{ }^{10} \text {, quae, cum } \\
{[\mathrm{h}] \text { interpellasset eum de concubitu, }} \\
\text { reiecta amorem in iram contulit dixit- } \\
\text { que temptatam esse a Bellerophonte }{ }^{11} \text { de } \\
\text { stupro. Proetus }{ }^{12} \text { credens coniugi misit } \\
\text { illum ad Iobaten }{ }^{13} \text { socerum suum prae- } \\
\text { cepitque ut obi[e]ceretur ad Chim<a>e- } \\
\text { ram bestiam. quam Bellerophons supe- } \\
\text { ravit ex facili usus consilio. Item Isido- } \\
\text { rus de Chim<a>era (Isid. Etym. } 11.3 .36 \text { ): } \\
\text { 'Fingunt Chim<a>eram triformem be- } \\
\text { stiam: ore leo, postremis partibus draco, } \\
\text { media caprea. Quam quidam physiolo- } \\
\text { gi }{ }^{14} \text { non animal, sed Ciliciae }{ }^{15} \text { montem } \\
\text { esse dicunt, quibusdam locis leones et } \\
\text { capreas nutrientem, quibusdam arden- } \\
\text { tem, quibusdam plenum serpentibus. } \\
\text { Hunc Bellerophons }{ }^{16} \text { habitabilem fecit, } \\
\text { unde Chim<a>eram dicitur occidisse'. }\end{array}$ & quam bellerofontes occidit. \\
\hline \multicolumn{2}{|c|}{ 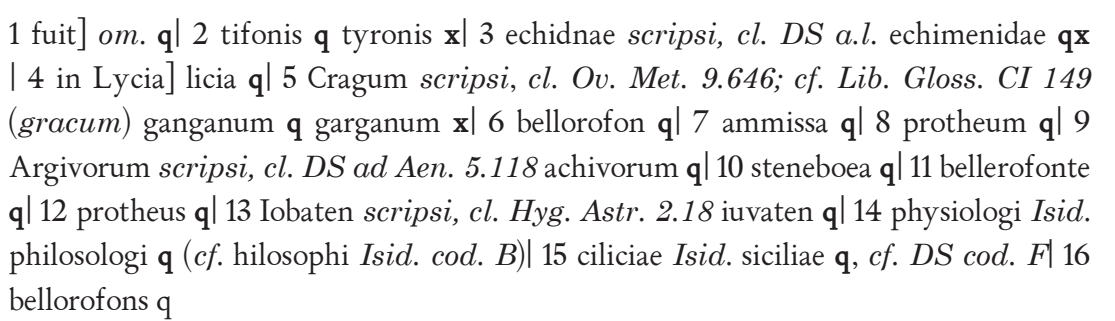 } \\
\hline
\end{tabular}


Un dato molto significativo è la coincidenza del testo di $\mathbf{x}$ con la voce chimaera del Liber Glossarum (Lib. Gloss. CI 149 = FL 138 ${ }^{30}$ ), la cui origine era già stata individuata in glosse virgiliane antiche perdute ${ }^{31}$. Tuttavia, alcuni elementi distinguono il testo di $\mathbf{x}$ da quello del Lib. Gloss., avvicinandolo invece allo scolio di q: oltre a condividere due varianti (echimenidae per Echidnae e garganum/ganganum per Cragum), entrambi i manoscritti riportano la notizia genealogica di matrice esiodea sulla Chimera (Typhonis et Echidnae filia, cf. Hes. Theog. 319). L'inciso, omesso dal Liber Glossarum, coincide con l'aggiunta DS alla nota serviana a.l.:

Serv. ad Aen. 6.288 flammisque armata Chimaera Typhonis et Echidnae filia, ore leo, postremis partibus draco, media caprea secundum fabulas fuit. re vera autem mons est Ciliciae, cuius hodieque ardet cacumen, iuxta quod sunt leones; media autem pascua sunt, quae capreis abundant, ima vero montis serpentibus plena. hunc Bellerophontes habitabilem fecit, unde Chimaeram dicitur occidisse.

Tuttavia, la descrizione della Chimera e soprattutto l'informazione relativa alla Licia e al monte Crago in Licia non derivano da questa nota del commento serviano: anzi, diversamente da tutti gli altri autori che trattano l'argomento (e.g. Plin. nat. 2.236; Sol. 39.1), Servio colloca il mostro in Cilicia piuttosto che in Licia ${ }^{32}$. Si può invece osservare che il passo di Isidoro citato nello scolio di q dipenda proprio da Serv. ad Aen. 6.288, combinato forse con qualche altra fonte (onde la dicitura quidam physiologi: la versione seguita da Servio si ritrova, ad esempio, anche in Palaeph. 28). Un dato ancor più specifico è costituito dal nome del monte licio (Kpóyos, Cragus) in prossimità del quale si trovava la Chimera; eccone le altre attestazioni:

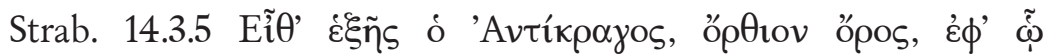

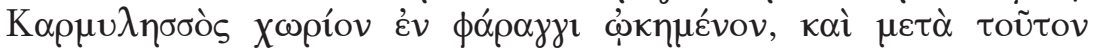

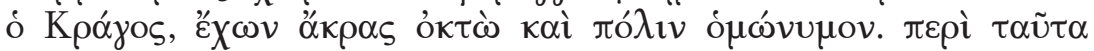

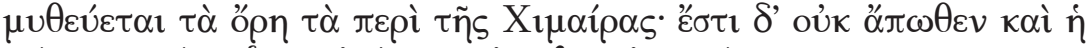

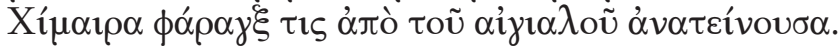

${ }^{30}$ Ecco il testo di CI 149 con le varianti di FL 138 secondo l'ed. Lindsay (Glossaria Latina, edd. W. M. Lindsay, J. F. Mountford, J. Whatmough, I, Paris 1926): monstrum fuit quod primas partes leonis, sequentes caprae (-preae FL 138) vomentis ignem, ultimas in cauda serpentis habuit. De qua Vergilius 'flammisque armata Chimaera'(de qua... Chimaera om. FL 138). Haec in Lycia iuxta Gracum (graecum FL 138) montem populabatur terras.

${ }^{31}$ Il Liber Glossarum rimonterebbe a questa fonte tramite il glossario Abstrusa (in una forma più estesa rispetto a quella attuale) secondo W. M. Lindsay, M. J. Thomson, Ancient Lore in Medieval Latin Glossaries, London 1921, 55, 180.

${ }^{32}$ Perciò lo Stephanus correggeva Ciliciae in Lyciae; tuttavia l'errore di Servio è confermato da Isidoro. 
Ov. met. 9.646-8

iam Cragon et Limyren Xanthique reliquerat undas, quoque Chimaera iugo mediis in partibus ignem, pectus et ora leae, caudam serpentis habebat.

Porph. ad Hor. carm. 1.21.8 Gragus mons Lyciae, in quo Chimaera fuisse dicitur.

Esaminando il passo di Ovidio si può notare anche una certa somiglianza con il testo del Liber Glossarum e lo scolio tràdito da $\mathbf{x}$ (ultimas in cauda serpentis habuit caudam serpentis habebat). Servio invece segue più da vicino la famosa ripresa omerica di Lucrezio:

Lucr. 5.904-6

qui fieri potuit, triplici cum corpore ut una,

prima leo, postrema draco, media ipsa, Chimaera

ore foras acrem flaret de corpore flammam?

Infatti, il criterio secondo cui Servio elenca le parti di cui consta il corpo del mostro (prima le parti estreme, poi quella centrale: leone, serpente, capra) è lo stesso di Lucrezio (cf. Hom. Il. 6.1819); invece lo scolio di qx ripristina la successione normale (leone, capra, serpente), che del resto figurava già in Esiodo (Theog. 322).

Per completare il quadro, resta da esaminare il resto dello scolio tràdito dal solo q: la parte su Bellerofonte (Bellerophons ${ }^{33}$ autem...usus consilio) dipende principalmente da una nota di commento del Servius Auctus:

DS ad Aen. 5.118 CHIMAERAM fabula ex qua hoc nomen translatum est talis est. Bellerofontes (Belleroph- F) admissa per ignorantiam caede, profugit ad Proetum Argivorum regem. a quo cum esset susceptus hospitio a Stheneboea uxore eius adamatus et de stupro interpellatus est. qui cum frequentius hoc se indicaturum Proeto minaretur, praeoccupatus a Stheneboea ipse apud Proetum accusatus est, tamquam reginae castitatem adpetisset. quem cum Proetus ad Iobatem (iovaten $\mathrm{F}$ iovatem $\mathrm{T})^{34}$, Lyciae regem, socerum suum cum litteris signatis, quibus praecipiebat ut puniretur, misisset, Iobates (iovates T iovatis F) Bellerofontem (BellerophF) adversus †tympios sibi Solymos, gentem ferocissimam misit.

${ }^{33} \mathrm{Nel}$ testo dello scolio tràdito da $\mathbf{q}$ ho uniformato la grafia del nome di Bellerofonte perché in un caso (quam Bellerophons superavit) q conserva la forma corretta. Ho invece lasciato invariata la forma bellerofontes nel testo di $\mathbf{x}$ (cf. DS a.1.).

${ }^{34}$ Iobatem (come Iobates poco dopo) è congettura del Masvicius. 
quos cum ille superasset, revertens in insidias quas sibi Iobates struxerat incidit, quibus prostratis novissime missus adversus Chimaeram, triplex monstrum, siquidem prima pars eius leo erat, posterior draco, in medio caput caprae, quod ignes efflabat. hanc ille vectus Pegaso equo qui volabat, occidit. miratus hanc eius constantiam Iobates (iovatis FT) filiam suam ei cum parte regni in matrimonium dedit.

Si può subito notare che anche la descrizione della Chimera contenuta nella prima parte dello scolio di $\mathrm{q}$ risente molto di questo testo (triplex... forma triplex monstrum; prima facie prima pars; flagrabat igni ignes efflabat): perciò credo che la prima parte dello scolio tràdito da $\mathbf{q}$ sia il risultato della contaminazione tra uno scolio simile a quello di $\mathbf{x}$ e il testo di DS ad Aen. 5.118.

Per quanto riguarda il mito di Bellerofonte, le coincidenze con il Servius auctus sono evidentemente vistose e richiamano la fattura di altri scolii di qx (come quello relativo a Teucro): ragion per cui, sebbene questa parte sia assente in $\mathbf{x}$, è probabile che la sua redazione sia comunque contestuale ad altri scolii comuni ai due manoscritt ${ }^{35}$. Ciò risulta confermato anche dalla qualità del dettato e delle informazioni fornite: un particolare (ut obiceretur ad Chimaeram ut...obiceret Chimaerae) trova riscontro preciso in Igino astronomo ${ }^{36}$, che peraltro offre un parallelo interessante per la notizia relativa alla Licia (in Lycia...populabatur terras Lyciorum agros flamma vastabat).

Notevole somiglianza con il nostro scolio presenta anche stavolta il Mitografo Vaticano, sebbene il capitolo su Bellerofonte (I 70) dipenda principalmente dal commento di Lattanzio Placido a Stazio, Theb. 4.589 (a sua volta debitore di Igino). Infatti ritroviamo (sia in Lattanzio che nel Mitografo) un segmento testuale molto simile allo scolio di q (Proetus... misit illum ad Iobaten socerum suum Proetus vero ad Iobatem socerum suum misit eum), con la stessa corruzione del nome Iobates (iuvaten $\mathbf{q}$,

${ }^{35}$ In effetti, la breve conclusione dello scolio di $\mathbf{x}$ (quam bellerofontes occidit) sembra riassumere drasticamente il lungo scolio sopravvissuto in q. Inoltre, in corrispondenza di Aen. 5.118 (Chimaeram) $\mathbf{x}$ presenta una glossa (vergata da una mano differente rispetto a quella responsabile degli scolii $\mathbf{q x}$ ) che si avvicina alla rielaborazione di DS presente nella prima parte dello scolio tradito da q ad Aen. 6. 288; eccone il testo: chimaera monstrum est cuius prima pars leo, media caprea, postrema draco.

${ }^{36}$ Hyg. Astr. 2.18 (ed. Le Bœuffle) Alii dicunt, quo tempore Bellerophontes ad Proetum Abantis filium, Argivorum regem, devenerit, Antiam regis uxorem hospitis amore inductam, petisse ab eo uti sibi copiam faceret, promittens ei coniugis regnum. Quae, cum inpetrare non potuisset, verita ne se ad regem criminaretur, occupat: eum sibi uim adferre uoluisse Proeto dicit. Qui quod eum dilexerat, noluit ipse supplicium sumere, sed quod ei [Pegasum] equum esse sciebat, mittit eum ad Iobatem, Antiae patrem, quam alii Sthenoboeam dixerunt, ut ille filiae pudicitiam defendens, Bellerophontem obiceret Chimaerae, quae eo tempore Lyciorum agros flamma vastabat. 
iuvatem Lact. Plac., Myth. Vat., cf. iovates DS). Significative sono anche altre coincidenze (de concubitu ut secum concumberet; temptatam esse a Bellerofonte de stupro ab eo se compellatam fuisse; il Mitografo dopo compellatam aggiunge pro stupro). Il capitolo successivo del Mitografo presenta ancor più forti analogie con lo scolio tràdito da $\mathbf{q}$; si veda il testo edito da Zorzetti:

Myth. Vat. I 71 Chimera monstrum, Tyronis ${ }^{37}$ et Achemenidae ${ }^{38}$ filia fuit. Cuius triplex dicitur forma: prima namque leonis horrebat facie, media caprae, cauda imitabatur ${ }^{39}$ draconem. Haec cum in Lycia iuxta Gargarum ${ }^{40}$ montem popularetur terras, a Bellerophonte interfecta est. Quidam Chimaeram dicunt non animal, sed montem Lyciae, quibusdam locis leones et capreas nutrientem, quibusdam ard[er]entem, quibusdam plenum serpentibus. Hunc Bellorophons habita<bi>lem fecit, unde Chimaeram occidisse dicitur.

A quanto pare il Mythographus Vaticanus mutua l'excerptum di Isidoro (tacendo l'autore) direttamente dallo scolio virgiliano utilizzato come fonte per questo passo ${ }^{41}$, che anche in questo caso (come per lo scolio su Teucro) si avvicina più alla versione testimoniata da $\mathbf{q}$ che al testo di $\mathbf{x}$.

In base a quanto detto finora, sarei incline a ricostruire una parentela di questo tipo tra i vari testimoni chiamati in causa:

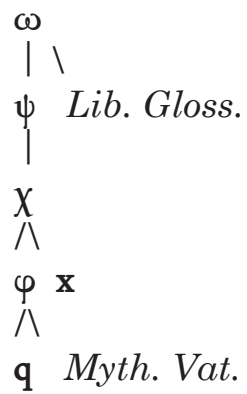

37 In apparato Zorzetti (Le premier mythographe, 44) annota: «scil. corruptum e typhonis». Si noti che la stessa corruzione si ritrova in $\mathbf{x}$ (ma potrebbe essere poligenetica).

${ }^{38}$ Zorzetti (Le premier mythographe, 44) in app.: «scil. corruptum ex aechidnae, cf. Hyg. fab. 151; Serv. auct. Aen. 6.288». Forse il Mitografo potrebbe aver corretto un originario echemenidae (qx) pensando al nome Achaemenides.

${ }^{39}$ Imitabatur è correzione di Mai per il tràdito minabatur, lezione che tuttavia trova conferma nello scolio di q. Piuttosto, nel testo del Mythographus sarebbe forse opportuno correggere draconem in draconis (cf. leonis, caprae).

${ }_{40}$ Zorzetti (Le premier mythographe, 43) pensa che iuxta Gargarum montem sia una "glose erronée» dovuta forse ad un travisamento di Verg. georg.1.103-04 (nullo tantum se Mysia cultu iactat et ipsa suas mirentur Gargara messes). Si tratta invece, più semplicemente, di una corruzione di Gragum (da Cragum).

${ }^{41}$ La variante Lyciae per Ciliciae è probabilmente congetturale. 
Secondo la mia ricostruzione, il testo di $\mathbf{x}$ ci restituirebbe più fedelmente (come mostra lo coincidenza con il Liber Glossarum) lo scolio virgiliano non serviano più antico, in seguito (probabilmente già nello stadio $X$ ) modificato con ricorso alle aggiunte DS e arricchito della digressione mitologica su Bellerofonte (con ricorso a DS e a Lattanzio Placido), nonché della citazione da Isidoro: il testo che ne risulta potrebbe esser confluito in un esemplare intermedio $(\varphi)$, da cui discenderebbero $\mathbf{q}$ e il Mitografo.

In questo modo si spiegherebbe la diffrazione della lezione autentica Cragum, corrottasi in gracum (Liber Glossarum) e in gragum (stadio $\psi$, cf. Porph. ad Hor. carm. 1.21.8), indi in una forma (gargarum? stadio $X$ ) che ha dato origine alle varianti garganum (x), ganganum (q), gargarum (Myth. Vat.), probabilmente anche per influsso dei nomi di altre cime più note (il Gargaro e il Gargano). Anche altre varianti sembrano confermare la validità dello schema tracciato: così nello scolio ad Aen. 1.619 compulerat si sarebbe corrotto in compellerat (stadio $\mathrm{X}$, da cui $\mathbf{x}, \varphi$ ), corretto da $\mathbf{q}$ in compellebat e sostituito dal Mitografo con subegerat. Allo stadio $\chi$ risalgono inoltre altri errori (salamoniam, echimenidae) condivisi da qx e/o dal Mitografo: è in questa fase della tradizione (e nello stadio $\varphi$, ad esso strettamente connesso) che gli scolii $\mathbf{q x}$ avrebbero assunto la loro fisionomia attuale, tipica dei codici virgiliani di età carolingia, come dimostra la stessa citazione puntuale di Isidoro nello scolio appena esaminato ${ }^{42}$; peraltro lo stesso passo di Isidoro è accostato alla glossa virgiliana già nel Liber Glossarum (cf. CI 148).

Naturalmente si tratta di un'ipotesi esplorativa, che andrà confermata sulla base di dati più completi (specie per quanto riguarda x e il Liber Glossarum); allo stato attuale delle conoscenze si potrebbero immaginare anche altri scenari che io non ho intravisto oppure ho giudicato meno probabili ${ }^{43}$.

Tornando a Isidoro, l'esempio della Chimera non è isolato: anche altri scolii tràditi da $\mathbf{q}$ e/o $\mathbf{x}$ contengono del materiale tratto dalle Etymologiae, sebbene in genere la citazione non sia così precisa ed esplicita. Per cominciare, esaminiamo uno scolio comune ad entrambi i manoscritti (anche se la versione di $\mathbf{x}$ è più breve):

${ }^{42} \mathrm{Si}$ veda ad esempio un altro manoscritto virgiliano d'età carolingia (Reg.lat. 1669, metà IX sec., Reims), dove il commento di Servio copiato nei margini è arricchito con scolii di provenienza diversa e con citazioni da Macrobio, Agostino e Isidoro: S. Ottaviano, "Il Reg. Lat. 1669: un'edizione di Virgilio d'età carolingia", in Miscellanea Bibliothecae Apostolicae Vaticanae (16), 2009, 259-324.

${ }^{43}$ Ad esempio, si potrebbe supporre che $\mathbf{x}$, $\mathbf{q}$ e il Mitografo discendano da un archetipo comune e che le divergenze di $\mathbf{x}$ vadano ascritte a collazione con il Liber Glossarum. Tuttavia, alcuni indizi (cf. l'aggiunta relativa a Salamina nello scolio a Aen.1.619) mi sembrano confermare l'antichità del testo conservato da $\mathbf{x}$. 


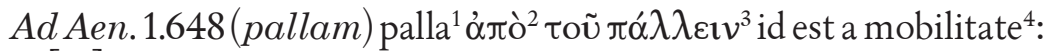
quadr[at]um pallium muliebris vestis ${ }^{5}$ deductum usque ad vestigia ${ }^{6}$, auro $^{7}$ et ordine gemmis ornatum.

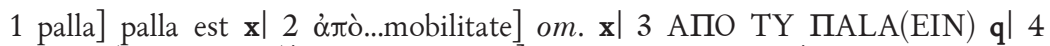
nobilitate $\mathbf{q}(=$ Isid. $\operatorname{codd}$.) $\mid 5$ muliebris vestis $] \mathbf{x}, c f$. Isid. muliebre $\mathbf{q} \mid 6$ usque ad vestigia deductum $\mathbf{x} \mid 7$ auro...ornatum] om. $\mathbf{x}$

Lo scolio è tratto da un passo di Isidoro (ed. Lindsay ${ }^{44}$ ):

Isid. Etym. 19.25.2 palla est quadrum pallium (palleum Isid. codd. $\left.C B Y^{2} X K M\right)$ muliebris vestis, deductum usque ad vestigia, quod [sic! quod del. Arev.] adfixis in ordinem gemmis. Et palla dicta ò

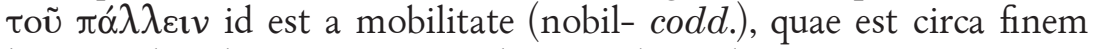
huiusmodi indumenti; sive quod rugis vibrantibus sinuata crispetur.

A sua volta, Isidoro aveva attinto al commento serviano relativo al passo in questione $\mathrm{e}^{45}$, ma di esso non vi è traccia nello scolio qx (dove infatti è riprodotto un errore del testo di Isidoro, nobilitate per mobilitate, conservato nella forma corretta dalla tradizione serviana). Lo stesso 'triangolo' testuale si verifica per altre glosse, stavolta tràdite dal solo q:

Ad Aen. 5.288-9 (theatri) $\mathrm{t}<\mathrm{h}>\mathrm{eat}[\mathrm{h}] \mathrm{rum}$ a spectando dictum quod ibi ludi aspiciuntur. Circus a circuitu equorum ubi currunt vel a Circe inventrice ${ }^{46}$.

Ad Aen. 7.741 (cat $[h] e<i>a s)$ cat $[\mathrm{h}] \mathrm{e}<\mathrm{i}>\mathrm{a}$ quam $<\mathrm{H}>$ oratius caiam dicit qualis fuit clava Herculis, cubitum et dimidium (demedium q) habens. est genus gallici teli cum magna materia et gravi<tate>. omne

${ }^{44}$ Per uniformità cito tutti i passi di Isidoro da: Isidori Hispalensis Etymologiarum sive originum libri XX, ed. W. M. Lindsay, Oxonii 1911. Talvolta ho segnalato delle varianti ricavandole da edizioni più recenti (in particolare, per il libro 19: Isidoro de Sevilla, Etimologías, Libro XIX: De naves, edificios y vestidos. Introducción, edición crítica, traducción y notas por Miguel Rodríguez-Pantoja, Paris-Córdoba 1995). Sarebbe utile studiare sistematicamente la tradizione testuale di Isidoro nei casi di accordo con gli scolii $\mathbf{q x}$, ma si tratta di un'indagine che esula dagli scopi limitati di questo lavoro.

${ }^{45}$ Serv. ad Aen. 1.648 «PALlam Rigentem duram propter aurum, sicut vel novas vestes videmus. significat autem tunicopallium, quod secundum Varronem palla dicta est ab

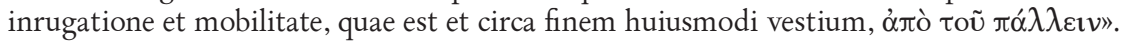

${ }^{46} \mathrm{Cf}$. Isid. Etym.15.2.34 circum Romani dictum putant a circuitu equorum, eo quod ibi

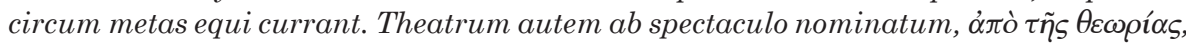
quod in eo populus stans desuper atque spectans ludos scaenicos contemplaretur; Serv. ad Aen. 5.288 "MEDiaqve IN VAlLE THEATRI media in valle erat circus theatri, id est spatium spectaculi. et 'theatri' Graece dixit a circumspectione. omne spectaculum theatrum

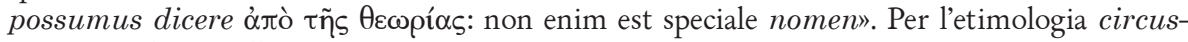
Circe si veda Tertull. spect. 8 . 
quod tangit ictu frangit $\langle$ nec $>$ longe evolat de muro et de alto loco tor<que>tur. alio nomine teutonas (tautanos Isid., teutonos Isidori codd. XYc, cf. Verg. Aen. 7.741 Teutonico ritu) dicunt quia in Teutonia Germaniae provincia antiquitus in usu habebantur ${ }^{47}$.

A parte Isidoro, negli scolii comuni a $\mathbf{q x}$ si trovano altri esempi di auctoritates esplicitamente nominate; ciò accade ad esempio per alcune glosse proprie del solo q, dove tuttavia il riferimento non è accompagnato dalla citazione estesa; in questi casi la scelta degli autori si rivela meno scontata e pone persino qualche problema d'identificazione. Ecco le glosse in questione:

Ad Aen. 1.652 (Laedae) uxoris Terei regis quam violavit Iovis mutatus (mot- q) in cycnum (cicuum q), ut in <carmine> Clementis dicitur.

Qui il riferimento probabilmente è a Prudenzio (Aurelius Prudentius Clemens), Peristeph. 10.221 (cycnus stuprator); una glossa simile si ritrova in un manoscritto dell'XI sec. di origine anglosassone ${ }^{48}$. Un altro caso è il seguente:

Ad Aen. 3.360 Clari id est Apollinis qui est sol; quid enim clarius sole est? Columella (colomella q) autem dicit quod est oppidum Clarus nomine et corripitur prima syllaba. deinde dicitur A pollo Clar $<i>$ us qui ibi maxime adoratur.

Poiché non vi è traccia in Columella di alcuna menzione di Claro, tenderei ad ipotizzare una corruzione del nome: si può forse intendere Pomponio Mela, che effettivamente parla del santuario a Apollo a Claro (chor. 1.88)? La glossa è interessante anche in relazione al testo virgiliano, perché corrobora la possibilità che la lezione corretta a Aen. 3.360 sia Clari (da Claros, lezione

${ }^{47}$ Cf. Isid. Etym. 18.7.7 clava est qualis fuit Herculis, dicta quod sit clavis ferreis invicem religata; et est cubito semis facta in longitudine. Haec et cateia, quam Horatius caiam dicit. Est enim genus Gallici teli ex materia quam maxime lenta, quae iacta quidem non longe propter gravitatem evolat, sed quo pervenit, vi nimia perfringit; quod si ab artifice mittatur, rursum redit ad eum qui misit. Huic [sic!] meminit Vergilius dicens (Aen. 7.741) 'Teutonico ritu soliti torquere cateias'. Unde et eos Hispani et Galli tautanos vocant; Serv. ad Aen. 7.741 "CATEIAS tela Gallica: unde et Teutonicum ritum dixit. cateiam quidam asserunt teli genus esse tale quale aclydes sunt, ex materia quam maxime lenta, cubitus longitudine, tota fere clavis ferreis illigata, quas in hostem iaculantes lineis, quibus eas adnexuerant, reciprocas faciebant. CATEIAS id est hastas. cateiae lingua Theotisca hastae dicuntur». Si confronti anche il Lib. Gloss. CA 988 (ricavato probabilmente da Isidoro).

${ }^{48}$ Iovem dicit qui versus in cignum ledam vitiavit: H. D. Meritt, The Old English Prudentius Glosses at Boulogne-sur-Mer, Stanford 1959. 
tràdita a testo dallo stesso q) e non Clarii (da Clarius $)^{49}$. Nulla di simile si trova né in $\mathbf{x}^{50}$ né in Servio ${ }^{51}$.

Infine, un riferimento a Gerolamo (a proposito del nome di Didone) si trova in una nota di $\mathrm{q}$ ad Aen. 4.335, purtroppo ormai quasi illegibile.

Un'altra fonte impiegata nelle glosse di q, sebbene senza menzione esplicita, è un autore molto letto nel Medioevo, Darete Frigio; dall'Historia De Excidio Troiae $(6.13)^{52}$ deriva con buona probabilità il seguente scolio tràdito dal solo q:

Ad Aen. 3.351 (Scaeaeque...portae) Sc[h] eaeus nomen Neptuno est unde Sceae portae dicuntur. Portae troiae VI sunt id est: Antenorea (Antenorida q), Dardani[d]a, Ilia, Sc[h]<a>ea, Thymbraea (Tymbria q), Troiana.

Alle porte Scee si riferisce anche un altro scolio presente sia in $\mathbf{q}$ che in $\mathbf{x}$, sebbene in una forma alquanto diversa; anche stavolta ci troviamo di fronte alla menzione assai problematica di un'auctoritas (Cicerone: si veda la versione più ampia tràdita $\mathrm{da} \mathbf{x}$ ):

\begin{tabular}{|l|l|}
\hline \multicolumn{1}{|c|}{$\mathrm{q}$} & \multicolumn{1}{|c|}{$\mathrm{x}$} \\
\hline $\begin{array}{l}\text { Ad Aen. 2.612 (scaeas) sceleratas } \\
\text { quia per eas equ<u>s intravit vel scaeas } \\
\dagger \text { AחO TOY CKAIN† quia ab antiqui- } \\
\text { tate, id est antiquas portas. }\end{array}$ & $\begin{array}{l}\text { Ad Aen. 2.612 scaeas nomen portarum } \\
\text { per quas vectus equus vel portae mali } \\
\text { hominis (homines } \mathrm{x}) \text { dictae. Sceae portae } \\
\text { id est priscae portae quia Cicero dixit †apo } \\
\text { tu sceon } \dagger \text { hoc est ab his priscis. et ideo } \\
\text { priscae dicuntur quia primitus de portis } \\
\text { civitatis factae sunt. }\end{array}$ \\
\hline
\end{tabular}

La parte più incomprensibile dello scolio è l'etimologia di scaeus, che in qualche modo (ma mi sfugge come!) dovrebbe richiamarne l'antichità; la stessa glossa si ritrova anche nelle aggiunte DS a.l. («SCAEAs: priscas»), senza tuttavia ulteriori chiarimenti. Le diverse spiegazioni possibili del significato dell'aggettivo (fra cui anche quella alternativa prospettata dallo stesso scolio

${ }^{49}$ Si veda quanto ho argomentato in: "Nota a Aen. 3, 360", Materiali e Discussioni per l'Analisi dei Testi Classici 62, 2009, 65-71.

${ }^{50}$ Ecco la glossa di x relativa a Aen. 3.360 (f. 86v): tripodas id est altaria tres pedes habentia. clarii laurus Clarus nomen insulae a qua Clarius Apollo nominatus est.

${ }^{51}$ Serv. ad Aen. 3.360 "CLARII Lavrvs et hic 'qui sentis' subaudis. et Clarium oppidum est in finibus Colophoniorum, ubi Apollo colitur: unde Apollo Clarius. sane quaeritur, utrum 'Clarii' geminandum sit 'i'. et sciendum aliter non procedere: nam 'cla' brevis invenitur, ut Statius "nec Clarias hac luce fores Didymaeaque quisquam"».

${ }^{52}$ Dar. Phryg. De ex. Troiae 4.13 (ed. Meister) Ilio portas fecit, quarum nomina sunt haec: Antenorea (antenoridas cod. M), Dardania (dardanidas cod. M), Ilia, Scaea (scea cod. M), Thymbraea (timbria cod. M), Troiana. 
$\mathbf{q x}$ ) ricorrono con poche varianti in altre fonti (Ap. Soph. 142.9; schol. D in Il. 3.145; Eust. in Il. 1.621.4 Valk; Serv. ad Aen. 3.351), ma senza che mai si parli di antichità. Forse l'identificazione delle porte Dardanie con le porte Scee (schol. A in Il. 6.237b) potrebbe spiegare perché nello scolio queste porte siano considerate le prime costruite a Troia (Dardano era molto antico e Ilo

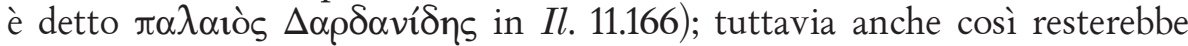
oscura l'etimologia fornita. Nello scolio $\mathbf{x}$ risultano poco perspicue anche le parole portae mali homines dictae, dove credo che homines vada corretto in hominis: si rimanda forse a Laomedonte, che fu sepolto in prossimità della porta Scea (Serv. ad Aen. 2. 241, 3.351)? In ogni caso, stavolta la connessione etimologica è evidente (malus okaı́́s scaevus, cf. sceleratas).

La materia trattata da Darete Frigio suscitava indubbiamente interesse per il compilatore dei nostri scolii: ad una particolare versione dei fatti relativi alla caduta di Troia rinvia un'altra annotazione comune a $\mathbf{q x}$, in cui si nomina un'auctoritas non altrimenti nota:

Ad Aen. 2.58: Pastores Dardanidae ${ }^{1}$ †Linchis $^{2}$ in libro $\mathrm{III}^{3}$ Aeneidos $†$ ab Aenea et Antenore exploratoribus captum Sinonem et ${ }^{4}$ ad Priamum adductum et non a pastoribus; quod declinavit Virgilius ne Aenean proditionis insimulare videretur, quem ${ }^{5}$ propter Augustum magis praedicare quam destruere ${ }^{6}$ debet.

1 Dardanidae om. $\mathbf{q} \mid 2$ Linchius $\mathbf{x} \mid 3$ tertio $\mathbf{x} \mid 4$ et $]$ om. $\mathbf{x} \mid 5$ quia $\mathbf{q} \mid 6$ vituperare $\mathbf{q}$

Lo scolio fa riferimento al tradimento di Enea e Antenore, una variante del mito conservata nella narrazione di Darete Frigio ${ }^{53}$, a cui allude anche Livio nel proemio della sua opera, sebbene nessuna delle fonti a noi note parli della cattura di Sinone da parte dei due eroi. Le testimonianze riguardanti il tradimento del solo Antenore sono più numerose (Lycophr. Alex. 340 ss. e sch. a.l.; Serv. ad Aen. 2.15) ed è stato anche supposto che nell'Ilias parva di Lesche il suo intervento fosse in qualche modo coordinato con la fuga di Sinone ${ }^{54}$. Questi, infatti, appare raffigurato nelle vesti di prigioniero (e non di supplice, come in Tryphiod. 263 ss., Quint. Smyrn. 12.360 ss.) nella Tabula Iliaca Capitolina (IG XIV 1284), ispirata proprio all'Ilias parva ${ }^{55}$. In effetti, Lesche probabilmente costituisce il precedente letterario seguito da Virgilio per modellare la figura di Sinone ${ }^{56}$.

${ }^{53}$ Dar. Phryg. De ex. Troiae 39-40.

${ }^{54}$ O. Immisch, ,Sinon' in Ausführliches Lexikon der griechischen und römischen Mythologie, Leipzig 1884-1937, rist. Olms, Hildesheim 1977-1978, IV, 939.

${ }^{55} \mathrm{Si}$ veda M. L. West, The Epic Cycle. A Commentary on the Lost Troy Epics, Oxford 2013, 165-6.

${ }^{56}$ R. Heinze, La tecnica epica di Virgilio, Bologna 1996, 37; N. Horsfall, Virgil, Aeneid 2: A Commentary, Leiden/Boston 2008, 587-91. Forse l'argomento era trattato anche da 
Sulla base di questo ragionamento si potrebbe tentare, in via meramente ipotetica, di scrivere Lesches in libro III Iliados in luogo del tràdito Linchis in libro III Aeneidos. In effetti non stupirebbe che il compilatore degli scolii possa esser incorso in una confusione onomastica così marcata, specie trattandosi di un nome greco a lui probabilmente sconosciuto; tuttavia, gli indizi e le conoscenze a disposizione sul poema di Lesche sono troppo scarni per fornire prove plausibili; anzi, sappiamo che nel seguito della storia nell' Ilias parva Enea veniva catturato da parte dei Greci (fr. 30 West), un particolare difficilmente conciliabile con la versione riportata dallo scolio circa il tradimento dell'eroe troiano.

Purtroppo né il confronto con Servio né con altri testi come il Mythographus Vaticanus ci conforta in alcun modo per la soluzione di questo enigma: sarei però propensa a considerare la fonte dello scolio antica e affine a quella delle aggiunte DS, sia per la formulazione della nota, sia per la precisione della citazione, sia per la natura stessa dell'argomentazione ${ }^{57}$, che sembra anche implicare una precedenza cronologica dell'autore citato rispetto a Virgilio.

In definitiva, anche in questo caso, come per il precedente, lo scolio lascia delle questioni in sospeso che forse potranno chiarirsi in futuro, grazie sia al contributo di altri studiosi, sia ad uno studio più approfondito della ricezione virgiliana negli autori e nei manoscritti d'età carolingia.

Tra gli scolii comuni a $\mathbf{q x}$ che, pur presentando qualche affinità con Servio, non sembrano esserne derivati, un buon numero si concentra nel sesto libro (per contenerli $\mathbf{x}$ aggiunge addirittura un foglio separato, il f. 120), dove evidentemente la ricorrenza di personaggi storici e mitologici deve aver stimolato l'attività esegetica. A titolo esemplificativo riporto uno di tali scolii:

una tragedia di Sofocle, il Sinon, ispirato anch'essa alla Piccola Iliade (S. Radt, Tragicorum graecorum fragmenta, Göttingen 1977, IV, 413-14).

${ }^{57}$ Spesso Servio nota, non senza una vena polemica, un intento adulatorio di Virgilio nei confronti di Augusto: Servius. Commentaire sur l'Énéide de Virgile. Livre VI, ed. E. JaunetMancy, Paris 2012, LXIX ss. 


\begin{tabular}{|c|c|}
\hline $\mathrm{q}$ & $\mathrm{x}$ \\
\hline $\begin{array}{l}\text { Ad Aen. 6, } 817 \text { animamque super- } \\
\text { bam: Brutus primus apud Romanos } \\
\text { consul factus est post Tarquinios regno } \\
\text { pulsos, qui ad Porsennam regem Etru- } \\
\text { riae venera<n>t. Porsenna autem legatos } \\
\text { ad Romanos misit bellum denuntians } \\
\text { (detutians q) nisi recepissent (recip- q) } \\
\text { Tarquinios. set Romani maluerunt bel- } \\
\text { lum gerere quam reges recipere illos. } \\
\text { quorum consilium Bruti filii scindere } \\
\text { voluerunt. promis[s]erunt legatis ut } \\
\text { nocte Tarquin[n]ios reciperent. hanc eo- } \\
\text { rum prodidit servus cogitationem patri. } \\
\text { unde pater iratus eos virgis c<a>e[s]sos } \\
\text { postea securi percussit. }\end{array}$ & $\begin{array}{l}\text { Ad Aen. 6, } 817 \text { ultoris Bruti: qui pri- } \\
\text { mus consul factus est, Brutus, Tarqui- } \\
\text { niorum scelus graviter ferens, detulit } \\
\text { ad populum querelam consuluitque } \\
\text { interficiendos esse tam nefandos reges } \\
\text { quos expulit populus Romanus. quos } \\
\text { recepit Porsenna misitque legatos ad } \\
\text { Romanos bella denuntians nisi recepis- } \\
\text { sent Tarquinios. Romani autem malue- } \\
\text { runt bellum gerere quam eos recipere. } \\
\text { quorum consilium filii Bruti scindere } \\
\text { voluerunt idest ut pelleretur consulatus } \\
\text { et ad se regiam potestatem revocarent. } \\
\text { promiserunt namque legatis ut nocte } \\
\text { Tarquinios reciperent. hanc cogitatio- } \\
\text { nem serv[i]us prodidit patri unde pater } \\
\text { eos virgis caesos postea securi percussit. }\end{array}$ \\
\hline
\end{tabular}

La nota serviana a.l. è più lunga e complessa: a partire dalle angherie di Tarquinio il Superbo si ripercorre la storia di Bruto fino al tradimento dei figli. A questo proposito Servio afferma: «Bruti filii amici erant filiis Tarquinii: cum quibus cum inissent consilium, ut eos per noctem intromitterent, proditi sunt a servo Vindicio et a patre interempti».

Nonostante qualche punto di contatto, colpiscono le differenze: in particolare, mentre Servio afferma semplicemente che Bruto uccise i suoi figli, nello scolio qx si descrive la dura punizione che inflisse loro. Particolarmente interessante è l'affinità con un passo dello ps. Aurelius Victor, De viris illustribus liber 10.5.33: quibus [scil. Tarquiniis] in exilium actis primus consul creatus filios suos, quod cum Aquiliis et Vitelliis ad recipiendos in urbem Tarquinios coniurarant, virgis caesos securi percussit. Si noti che il segmento testuale comune ai due passi (virgis caesos securi percussit) ricorre anche in un passo di Valerio Massimo ${ }^{58}$, e probabilmente si trovava in una fonte comune.

In conclusione, dai frammenti del perduto testimone virgiliano $\mathbf{q}$ emergono novità interessanti soprattutto sotto il profilo della ricezione del testo di Virgilio, che dimostrano la circolazione nel IX sec. di materiali esegetici non direttamente ricavati dal commento serviano e probabilmente in parte antichi e analoghi a quelli consultati dal compilatore del cosiddetto

58 Val. Max. 5.8.1 L. Brutus, gloria par Romulo, quia ille urbem, hic libertatem Romanam condidit, filios suos dominationem Tarquini a se expulsam reducentes summum imperium obtinens conprehensos proque tribunali uirgis caesos et ad palum religatos securi percuti iussit. 
Servius auctus. Scavi più approfonditi in tale direzione potranno contribuire a consolidare e arricchire questo nostro primo tentativo di ricostruzione. 